\title{
Hybrid closed Loop improved glucose control compared to sensor-augmented pumps in outpatients with type 1 diabetes in real-life conditions with telehealth monitoring
}

\author{
C. Thivolet $^{1}\left(\mathbb{1} \cdot\right.$ M. Gaudilliere $^{1} \cdot$ S. Villar Fimbel ${ }^{1} \cdot$ N. Bendelac ${ }^{1} \cdot$ B. Mestre ${ }^{1} \cdot$ N. Protsenko ${ }^{1} \cdot$ S. Brunot $^{1} \cdot$ M. Nicolino $^{1}$
}

Received: 22 September 2021 / Accepted: 21 October 2021 / Published online: 1 November 2021

๑) Springer-Verlag Italia S.r.l., part of Springer Nature 2021

\begin{abstract}
Objectives The study aims at evaluating glucose metrics and HbA1C values after pump initiation in outpatient settings. Research design and methods This single center observational study enrolled 121 subjects with type 1 diabetes between September 2020 and May 2021 initiating sensor-augmented pump therapy with stand-alone CGM $(n=26)$ or pump users who only changed their device $(n=51)$, with predictive low glucose management $(n=8)$ or with Hybrid Closed Loop using Medtronic 780G $(n=36)$ systems. Changes in HbA1C levels and glucose metrics were analyzed after 3 months. All subjects received diabetes and carbohydrate-counting education if needed at time of initiation and were proposed a telehealth monitoring by a diabetic nurse educator.

Results There was no episodes of severe hypoglycemia or diabetic ketoacidosis nor serious pump-related adverse events despite outpatient model of care. While only 18/121 (14.8\%) participants reached initially the recommended HbA1C levels, 23/85 (27\%) in the conventional group and 33/36 (91\%) subjects in the Hybrid Closed Loop group reached target levels after 3 months of follow-up. Time in target range $3.9-10 \mathrm{mmol} / \mathrm{L}(70-180 \mathrm{mg} / \mathrm{dl})$ also improved and was optimal with closed loop with 30/36 (83\%) subjects with time in range above $70 \%$.

Conclusions Initiation of insulin pump therapy for outpatients is safe with a dedicated facility. Telehealth monitoring after outpatient initiation provides tools for improvement in glucose control with an insulin pump. Outpatient pump initiation is compatible with Hybrid Closed Loop systems which provide the largest improvements in glucose control.
\end{abstract}

Keywords Insulin pump · Outpatient · Type 1 diabetes · Telemedicine

\section{Introduction}

Achieving glycemic targets to prevent diabetic complications remains challenging [1]. This is clearly true for adolescents and young adults as indicated in several diabetes registries $[2,3]$. Insulin delivery administrated by pumps (continuous subcutaneous insulin infusion, CSII) has several advantages such as flexibility and reduction of severe hypoglycemia [4-6]. Use of this therapeutic option increased dramatically in the last decade [7]. A greater superiority of CSII over multiple daily injections (MDI) regimen was notably found

Managed by Massimo Porta .

C. Thivolet

charles.thivolet@chu-lyon.fr

1 DIAB-eCARE Diabetes Center, Hospices Civils de Lyon, Lyon, France in those subjects with the highest HbA1C levels at baseline [8]. Sensor-augmented pumps (SAP) with the addition of interstitial continuous glucose monitoring (CGM) gave additional insights in terms of adjustment to metabolic needs either as a stand-alone device $[9,10]$ or with predictive low glucose management (PLGM) as shown in randomized clinical trials (10). In addition, CGM has also help to define new glycemic targets for prevention of diabetic complications and hypoglycemia [11]. Hybrid Closed Loop (HCL) systems for people with type 1 diabetes have further improved diabetes control when compared to SAP [12]. Most of the studies that evaluate CSII, SAP or HCL therapies are trials that included selected populations of subjects with high levels of motivation that may not reflect the routine use of these therapies. In addition, modalities of reimbursement may vary across countries with involvement of private insurance payers which may increase social disparity. As with all new technology, it becomes increasingly important to translate 
in real-world situations the results from controlled studies with the required time to provide adequate education and follow-up. Modalities of CSII initiation differ according to centers and national regulations. In France, reimbursement is limited to inpatients from initiating centers [13]. Since the outbreak of the COVID-19, access to clinics dedicated to diabetes care has been limited during lockdown and activities were largely transitioned to a telehealth/telemedicine model of care. CSII initiation in outpatient settings actively challenges current health care organizations. Here, we report the glycemic and safety outcomes of outpatient pump initiation with telemonitoring that resulted in reduced $\mathrm{HbA1C}$ levels and increased times in range values.

\section{Research design and methods}

\section{Study design and participants}

The study was a single center open-label analysis of clinical outcomes after CSII initiation during free-living conditions after attending DIAB-eCARE (www.diab-ecare.fr) a T1D integrated practice unit of the University Hospital of Lyon (France) between September 2020 and May 2021. At enrollment, participants or parents gave written consent to participate and baseline data were collected. All device training was performed by a diabetes nurse educator during a single visit in addition to the settings of telehealth parameters. The procedure to initiate insulin pump therapy in outpatient settings was authorized and funded by the French Health Authority in contrast to current regulatory procedures [14]. Three categories of participants were identified: first pump users $(n=26)$ with stand-alone CGM, current CSII users changing their device for SAP with stand-alone CGM $(n=51)$ or SAP and predictive low glucose suspend (PLGS) capacities using Tandem t:slim X2 ${ }^{\mathrm{TM}}$ insulin pump with Basal-IQ ${ }^{\mathrm{TM}}$ and Dexcom G6® CGM $(n=8)$ and subjects with Hybrid Closed Loop (HCL) technology $(n=36)$ using the Medtronic 780G TM pump and Smartguard 3® with automated correction bolus. All subjects received fastacting insulin analogs with either insulin Aspart or Lispro according to their previous therapy. Participants had willingness and ability to adhere to the observatory and shared data to health care providers with their smartphone and/or with Diabnext uploader or through CareLink ${ }^{\mathrm{TM}}$ Personal (Medtronic) when appropriate. Participants were asked to download their pump daily the first week then twice-weekly thereafter. A diabetes nurse educator monitored glucose and insulin data remotely at least once weekly to allow for realtime assessment of diabetes control and safety. The diabetes team members were alerted via text messages in case of adverse hyperglycemic or hypoglycemic events. Age, sex, duration of diabetes, body mass index (BMI), HbA1C values were recorded as well as glucose metrics with an observation frame of two weeks.

\section{Closed loop system}

Participants $(n=36)$ used the AHCL system in real-life conditions with the MiniMed 780G insulin pump, the guardian CGM components (Guardian sensor 3 and Guardian link transmitter) and a specific blood glucose meter (AccuChek® Guide). A minimum of three blood glucose tests per day was recommended for calibration of the sensor in accordance with the labeling of the CGM device. After a wash-out period of 1-2 weeks, insulin-to-carbohydrate ratios were set for each subject by investigators and auto-mode was activated with an initial target glucose set point of $6 \mathrm{mmol} / \mathrm{L}$ $(110 \mathrm{mg} / \mathrm{dl})$ and an active insulin time of $2 \mathrm{~h}$. These set points were chosen to obtain improved glycemic control without increasing the risk for hypoglycemia. A temporary set point of $8.3 \mathrm{mmol} / \mathrm{L}(150 \mathrm{mg} / \mathrm{dl})$ was used for exercise. In case of excessive low glucose levels, the glucose set point was increased to $6.7 \mathrm{mmol} / \mathrm{L}(120 \mathrm{mg} / \mathrm{dL})$ with longer active insulin times to obtain less aggressive dosing.

\section{Study objectives}

The primary objective was to evaluate the mean change in HbA1c levels between initial visit and after 3 months of follow-up based on treatment effect. Secondary objectives were percentage of glucose values in target range of $3.9-10 \mathrm{mmol} / \mathrm{L}(70-180 \mathrm{mg} / \mathrm{dL}$ ), glycemic variability (mean amplitude of glycemic excursions of sensor glucose values). Additional objectives were to confirm safety by comparing the percentage of glucose values in the percentage of time spent $\leq 3.0 \mathrm{mmol} / \mathrm{L}(54 \mathrm{mg} / \mathrm{dL})$ and above $250 \mathrm{mg} / \mathrm{dl}$.

\section{Statistical methods}

We report mean (SEM) for normally distributed variables and median (IQR) for skewed variables. Categorical variables were summarized by frequencies. Comparisons between baseline characteristics and outcomes after 3 months at home were performed using two-sided paired Student's t-test for normally distributed outcomes or a Wilcoxon signedrank test for skewed outcomes. The overall differences in percentage TIR between periods were compared by paired t-test and a significance level of 0.025 (one-sided). Comparisons were performed with Chi-square correlations between delta Final-Initial HbA1C values and initial HbA1C levels with Pearson test according to Gaussian distribution. Due to the limited number of subjects with a PLGM system, we pooled these individuals with those with stand-alone CGM in the SAP group for comparison with the HCL group using unpaired t-test or Mann-Whitney when appropriate. All 
statistical analyses were performed using GraphPad Prism 7.05 software (San Diego, CA) and Medistica Pvalue.io available on https://www.pvalue.io/fr,

\section{Results}

\section{Patient characteristics and CGM use}

A total of 121 subjects (71 females) with mean age 32.0 [20.2; 41.4] years including 26 subjects aged $<18$ years, were enrolled. The median length of diagnosis was 18.0 $[9.00 ; 28.0]$ years. Baseline demographics of the three categories of participants are described in Table 1 . With respect to technology use, 77 subjects were on SAP with stand-alone CGM, eight participants had a SAP + LGSM system and 36 subjects used a HCL system. As shown in Table 1, no difference in age, BMI, gender, baseline $\mathrm{HbA1C}$ levels could be noticed between groups. No difference in age, BMI, duration of disease, median initial $\mathrm{HbA1C}$ values and percentage of glucose values in TIR was found between groups.

\section{Evolution of $\mathrm{HbA1C}$}

All groups improved their diabetes control (Fig. 1) but at different levels. A significant reduction was found both in those subjects on MDI who initiated pump therapy with $7.66 \pm 0.05 \%(60.2 \pm 1.9 \mathrm{mmol} / \mathrm{mol})$ vs. $8.27 \pm 0.26 \%$ $(66.76 \pm 2.8 \mathrm{mmol} / \mathrm{mol}), p<0.0001$, but also in CSII users who changed their pump model with stand-alone CGM or SAP with $7.5 \pm 0.05 \%(60.54 \pm 1.12 \mathrm{mmol} / \mathrm{mol})$ vs. $7.88 \pm 0.11 \%(62.4 \pm 0.98 \mathrm{mmol} / \mathrm{mol}), p<0.001$. During HCL, mean \pm SEM HbA1C levels were significantly reduced with $6.84 \pm 0.05 \%(51.24 \pm 0.61 \mathrm{mmol} / \mathrm{mol})$ vs. $7.66 \pm 0.1 \%(60.69 \pm 1.2 \mathrm{mmol} / \mathrm{mol}), p<0.0001$ and were lower than subjects with SAP $(p<0.0001)$ as shown in Table 2. At 3 months, we observed 23/85 (27\%) in the SAP group and $33 / 36(91 \%)$ subjects in the HCL group within the recommended $\mathrm{HbA} 1 \mathrm{C}$ levels according to age. Differences between final and initial HbA1C levels were inversely correlated to initial HbA1C levels (Fig. 2) for subjects in the HCL group (R coefficient [95\% CI] $=-0,8687[-0,9308$ to $-0,7581], p=0.0001$ ) and also for participants who

Table 1 Characteristics of outpatients with type 1 diabetes who initiated pump therapy (initiation, $n=26$ ) or changed their pump model (switch, $n=95$ ). Initiation was performed exclusively with sensor-

augmented pumps (SAP) and already pump users had either SAP or HCL system. Variables are indicated as median value [25; 75 QI]

\begin{tabular}{llllll}
\hline & Total $(n=121)$ & Initiation $(n=26)$ & Switch $(n=95)$ & SAP $(n=85)$ & HCL $(n=36)$ \\
\hline Age years, median [25-75 QI] & $32.0[20.2 ; 41.4]$ & $29.0[16.0 ; 40.8]$ & $34.0[25.0 ; 33.5]$ & $29.0[17.8 ; 40.5]$ & $38.5[25.9 ; 44.7]$ \\
Duration of T1D, median [25;75QI] & $18.0[9.00 ; 28.0]$ & $17.0[10.5 ; 21.5]$ & $20.0[9.00 ; 31.0]$ & $17.5[8.88 ; 24.2]$ & $23.0[12.0 ; 33.0]$ \\
BMI median [25;75QI] & $23.4[21.3 ; 26.9]$ & $24.5[22.3 ; 27.8]$ & $23.3[21.3 ; 26.7]$ & $23.2[21.4 ; 27.4]$ & $23.9[21.3 ; 26.5]$ \\
Females (\%) & $71(58.6 \%)$ & $18(69 \%)$ & $53(55.7 \%)$ & $51(59 \%)$ & $20(55 \%)$ \\
Initial HbA1C values (mmol/mol) & $63.34( \pm 1)$ & $66.76( \pm 2.8)$ & $62.4( \pm 0.98)$ & $62.47( \pm 1.15)$ & $60.69( \pm 1.2)$ \\
Initial HbA1C values $(\%)$ & $7.95( \pm 0.09)$ & $8.27( \pm 0.26)$ & $7.88( \pm 0.11)$ & $8.04( \pm 0.11)$ & $7.70( \pm 0.11)$ \\
\hline
\end{tabular}

Fig. 1 Evolution of $\mathrm{HbA} 1 \mathrm{C}$ levels at baseline (M0) and after 3 months (M3) for subjects with HCL (A) or SAP (B) systems with HCL (A) or SAP (B) systems
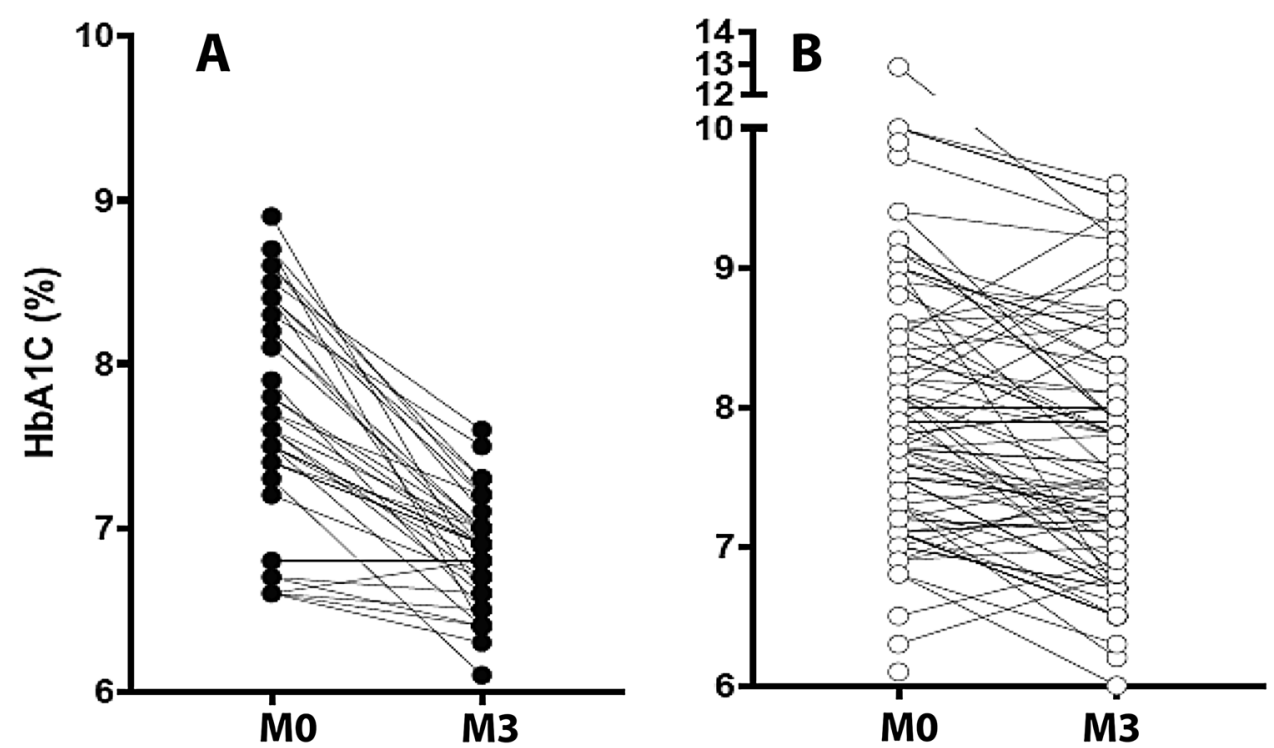
Table 2 Evolution of $\mathrm{HbA} 1 \mathrm{C}$ levels percentages of time in range (TIR) $3.9-10 \mathrm{mmol} / \mathrm{L}(70-180 \mathrm{mg} / \mathrm{dl})$ and coefficient of variation of glucose values between baseline at M0 and after 3 months of insulin pump therapy with sensor-augmented pumps (SAP) or Hybrid Closed Loop (HCL) systems

\begin{tabular}{lllr}
\hline & SAP $(n=85)$ & HCL $(n=36)$ & $p$ \\
\hline HbA1C MO, mean $( \pm$ SEM) $\% \mathrm{mmol} / \mathrm{mol}$ & $8.04( \pm 0.11) 64.56( \pm 1.10)$ & $7.70( \pm 0.11) 60.69( \pm 1.2)$ & 0.31 \\
HbA1C M3, mean $( \pm$ SEM) $\% \mathrm{mmol} / \mathrm{mol}$ & $7.66( \pm 0.1) 60.54( \pm 0.94)$ & $6.84( \pm 0.05) 51.24( \pm 0.61)$ & $<0.001$ \\
TIR M0, mean $( \pm$ SEM $)$ & $49.8( \pm 1.75)$ & $54.4( \pm 1.75)$ & 0.2 \\
TIR M3, mean $( \pm$ SEM) & $53.5( \pm 1.88)$ & $75.6( \pm 1.2)$ & $<0.001$ \\
CV M0, mean $( \pm$ SEM) & $41.0( \pm 7.27)$ & $37.7( \pm 1.17)$ & 0.031 \\
CV M3, mean $( \pm$ SEM) & $39.8( \pm 7.35)$ & $34.1( \pm 0.63)$ & $<0.001$ \\
\hline
\end{tabular}

Fig. 2 Correlations between initial $\mathrm{HbA1C}$ levels and delta values between initial and final HbA1C levels from subjects changing their pump for HCL (closed circles) or SAP (open squares) and those who initiated SAP (open circles). The broken line corresponds to the linear correlation for subjects with $\mathrm{HCL}$

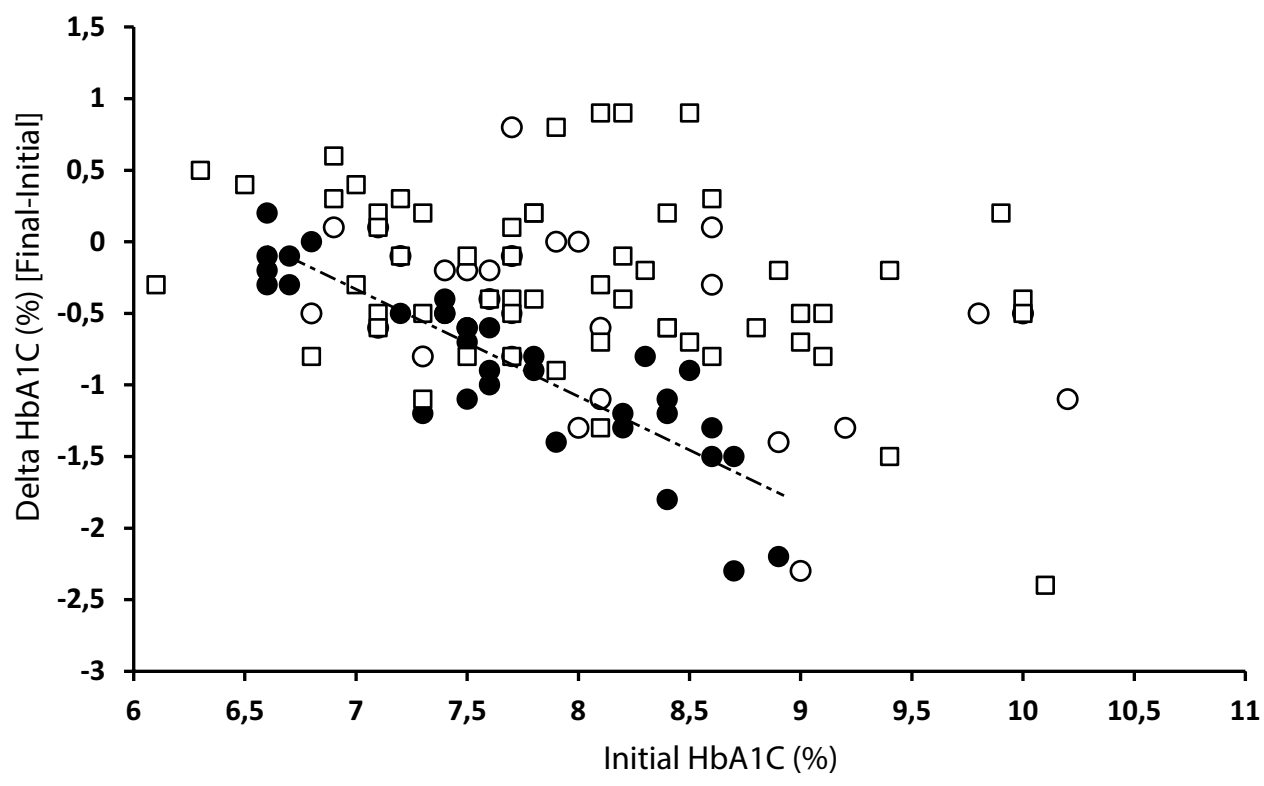

initiated SAP therapy $(\mathrm{R}$ coefficient $=-0,5131[-0,7564$ to $-0,1452], p=0.0073$ ) and for previously pump users on SAP with R coefficient of $-0,3081$ [ $-0,5303$ to $-0,04631]$ $(p=0.0186)$. The results were similar in adolescents $(n=26$, age $14[12 ; 16]$ years) with a mean reduction of $\mathrm{HbA1C}$ levels of $-0.6 \pm 0.9 \%$ from $8.15 \%$ [7.7;8.52] to $7.75 \%$ [7.2;8.12], $p<0.0001)$ including six subjects with HCL and a reduction of $-0.8 \pm 0.3 \%$ of $\mathrm{HbA1C}$ levels.

\section{Meeting glycemic targets}

A shown in Fig. 3, TIR increased both subjects with SAP or HCL systems. With respect to previous insulin experience, mean \pm SEM improvement in TIR $3.9-10 \mathrm{mmol} / \mathrm{L}$ (70-180 mg/dL) was similar between subjects who initiated or changed their pump with an open-loop SAP system $(57.64 \pm 3.36 \%$ vs. $53.46 \pm 1.8 \%, p=0.27)$. TIR was higher when HCL was used as compared to baseline $(75.57 \pm 1.2 \%$ vs. $49.8 \pm 1.76 \%, p<0.0001)$. The proportion of participants with TIR $\geq 70 \%$ during follow-up was $14 / 85$ (16.5\%) in the
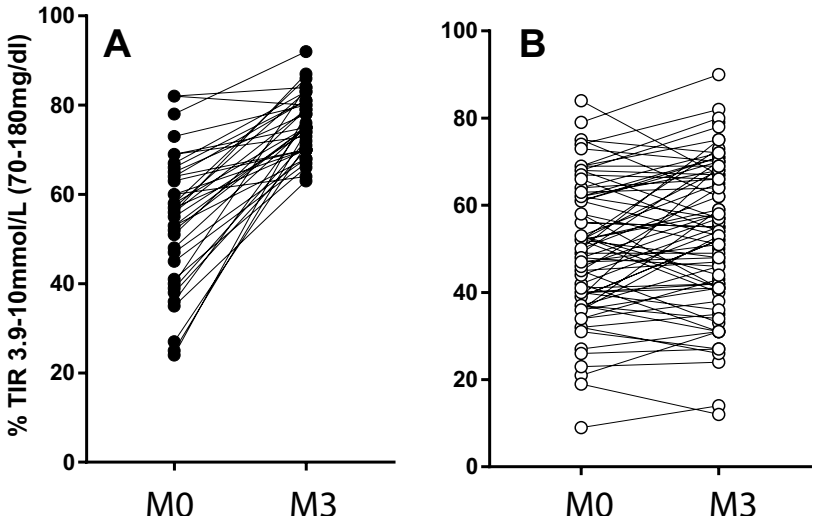

Fig. 3 Evolution of \% time in range at baseline (M0) and after 3 months (M3) of subjects with HCL (A) or SAP (B) systems

SAP group vs. 30/36 (83\%) in the HCL group. No significant difference was noticed at 3 months vs baseline in percentage of time $<3 \mathrm{mmol} / \mathrm{L}(54 \mathrm{mg} / \mathrm{dl})$ for HCL $(0.78 \pm 0.16 \%$ 
vs. $0.7 \pm 0.2 \%, p=0.46)$, first time SAP users $(0.9 \pm 0.32 \%$ vs. $0.75 \pm 0.28 \%, p=0.84$ ) or experienced users with SAP $(0.88 \pm 0.17 \%$ vs. $1.18 \pm 0.27 \%, p=0.44)$. At 3 months, the percentage of time between 3 and $3.9 \mathrm{mmol} / \mathrm{L}(54-70 \mathrm{mg} /$ dl) was lower during HCL vs. SAP with $2.08 \pm 0.26 \%$ and $3.36 \pm 0.28 \%$, respectively, $(p<0.001)$. HCL reduced significantly the time spent $>250 \mathrm{mg} / \mathrm{dl}$ in comparison to baseline $(4 \pm 0.49 \%$ vs. $14.5 \pm 1.74 \%, p<0.0001)$. This reduction was also seen in SAP group $(17.25 \pm 1.63 \%$ vs. $19.3 \pm 1.5 \%$, $p<0.001)$ but was limited. A significant reduction was observed in the number of participants who spent $<25 \%$ of time $>10.0 \mathrm{mmol} / \mathrm{L}(180 \mathrm{mg} / \mathrm{dL})$ after 3 months for subjects with HCL $(5 / 36$ vs. $25 / 36, p<0.0001)$ but not for SAP $(11 / 85$ vs. $15 / 85, p=0.52)$. A significant reduction in $\%$ $\mathrm{CV}$ was noticed in the HCL group with $34.1 \pm 0.63 \%$ vs. $37.7 \pm 1.17 \%(p<0.05)$, but not for subjects who initiated pump therapy or changed pump model in the SAP group.

\section{Discussion}

We provide the evidence that insulin pump initiation in outpatients is safe and efficient both for the initiation or change of pump model. This organization was designed to meet user's desire for convenience and improvement in diabetes management. This required the support of a dedicated health care team and telehealth monitoring. Metabolic improvement was heterogeneous but was maximal with Hybrid Closed Loop systems.

Although several guidelines have been published [16, 17], variation in insulin pump initiation regarding education and training modalities can be observed according to center's practice and national policies. Initiation of insulin pump therapy in an ambulatory setting is not new and has been used successfully in some centers. This requires flexible outpatient care program and 24-h health provider availability for assistance. Outpatient initiation of pump therapy has several advantages since patient's life can more quickly approach to normal with subjects able to work normally, go to school, and remain in their familiar surroundings. Several advantages for hospitalization have been advocated including stopping the long-acting insulin dose the night before pump initiation and progressive acquisition of knowledge with formal pump training sessions spanned during several days. Inpatient initiation is required in France for more than 20 years to get full coverage of patients by health insurance and to provide revenues for both the initiating center and health care providers who ensure the on-call and supply of patients at home [13]. This organization significantly increases the economic burden and has been seriously challenged by the COVID-19 pandemic with the dramatic reduction of hospitalization and educational capacities. The development of telemedicine and availability of connected devices allowed us to propose an alternative model for outpatients with an experimentation taken in charge by the payers [14].

Continuous subcutaneous insulin infusion (CSII) using an external pump is an intensive diabetes therapy recognized to improve metabolic control and to reduce both glycemic instability and frequency of severe hypoglycemia as shown in a meta-analysis of several studies [5]. CSII initiation requires to set basal rates, individualized insulin-to-carbohydrate ratios and sensitivity factors, a sum of educational and clinical factors that are more challenging to succeed in naïve individuals treated by MDI. CSII is also cost-effective vs. MDI across several settings for patients who have poor glycemic control and/or problematic hypoglycemia [18]. However, when mean $\mathrm{HbA1C}$ levels were analyzed in large clinic registries according to CGM and type of insulin administration, pump and injection users had similar results using CGM for glucose monitoring with the highest $\mathrm{HbA1C}$ levels in adolescent and young adults irrespective of device use [2]. While insulin pump and CGM use increased between 2010 and 2012 and 2016-2018, only a minority of adults and youth with T1D in the USA achieve ADA goals for $\mathrm{HbA} 1 \mathrm{c}$. This was also observed in our population with only $18 / 121(14.8 \%)$ participants who met initially the recommended $\mathrm{HbA} 1 \mathrm{C}$ targets of $\leq 7 \%$ for adults [11] or $7.5 \%$ for those before 18-year-old [15]. Addition of CGM for people managing their diabetes with an insulin pump has clear benefits as shown in the SWITCH crossover trial [9]. The ability of control devices to provide automatic flow of data to allow caregivers to remotely monitor insulin and glucose data provides additional efficiency as shown previously (19).

Development of insulin pump therapy has several strategic issues and management of diabetes is moving rapidly into the area of sensor-augmented therapy which now encompasses closed loop approaches. As insulin pump therapy is a large contributor to diabetes health care expenditures among insulin treated patients [20], it is urgent to optimize the metabolic results. Although many studies have demonstrated the cost-effectiveness of CSII over MDI including less acute events and hospitalizations, reduction of costs is a necessity with regard to new indications and solutions. This requires appropriate education and follow-up including different devices as well as data transfer and management. It is interesting that we observed significant improvements in all conditions, i.e., naïve vs experienced subjects. We observed significant improvements in $\mathrm{HbA} 1 \mathrm{C}$ values with a larger but still insufficient proportion of subjects meeting glycemic targets in those who changed their pump model. As expected, we were able to confirm the superiority of HCL vs SAP systems in the capacity to reach target glucose values. Beside the addition of automated insulin administration, it must be emphasized that HCL requires announced meals with carbohydrate counting which is not the case for SAP. 
Possible changes in diet profiles may occur according to pump systems but this has not yet been examined.

Changes in model of care cannot come at a cost of greater hypoglycemia. The percentage of time $<3 \mathrm{mmol} / \mathrm{L}(54 \mathrm{mg} /$ dl) and $<3.9 \mathrm{mmol} / \mathrm{L}(70 \mathrm{mg} / \mathrm{dl})$ was far below the recommendations, including additional benefits in the HCL group. The improvements observed in our study for patients with previous pump experience clearly underline the added value of carbohydrate counting [21] and telehealth monitoring as previously noticed by our group [19]. This also suggests that conditions for access and follow-up are also important factors for success. It cannot be excluded that our population was selected and strongly motivated to improve their glucose control due the simplified procedures and therefore may not reflect the general population of subjects with diabetes. Initiation of pump therapy in outpatients requires that health care professionals determine which patients are likely to benefit the most from CSII. This implies pre-pump start preparation and planning.

Insulin pump initiation in outpatient settings does not alter the performance of HCL system. Our results after 3 months in the real life confirm the superiority of this automatized insulin administration and the capacity to reach the recommended glucose values. Our data also provide confidence in the system's performance across different age groups including adolescents with similar results to randomized studies [22]. The capacity to provide continuous patient education with telemedicine both for pump parameters adjustments and carb counting issues are important factors for success in our outpatient model of care. In few subjects with SAP, telehealth monitoring was not possible due to technical reasons or was refused. This may explain in part the heterogeneity of the results in this group. The ability for caregivers to view patient data without manual uploads is still an unmet challenge due to the absence of automatic flow of data for all devices. The pace of development in diabetes technology is extremely rapid. It is mandatory that data could be automatically shared with secured cloud-based data management systems. During this process, the most important component remains the patient. It is therefore crucial to maintained dedicated teams with specific skills to assist the patient in device/ program selection and to support its use through ongoing education and training with both presential and virtual diabetes care.

In conclusions, we provide evidence for the safe combination of outpatient initiation of insulin pump with adapted education programs and telehealth monitoring performed by a dedicated healthcare team. This organization is perfectly adapted for the initiation of closed loop systems.

Acknowledgements We are indebted to patients who followed us in our new center, to nurses and dieticians involved in pump initiation. This experimentation is financed by the French social healthcare system through a special authorization from the Health Agency of Auvergne Rhone-Alpes Region (ARS AuRA).

\section{Declarations}

Conflict of interest They authors declare that they have no conflict of interest.

Ethical approval The study was a single center open-label analysis of clinical outcomes after CSII initiation during free-living conditions after attending DIAB-eCARE (www.diab-ecare.fr) a T1D integrated practice unit of the University Hospital of Lyon (France) between September 2020 and May 2021.

Informed consent At enrollment, participants or parents gave written consent to participate and baseline data were collected.

\section{References}

1. Diabetes Control and Complications Trial/Epidemiology of Diabetes Interventions and Complications (DCCT/EDIC) Research Group, Nathan DM, Zinman B, Cleary PA, et al. (2009) Modernday clinical course of type 1 diabetes mellitus after 30 years' duration: the diabetes control and complications trial/ epidemiology of diabetes interventions and complications and Pittsburgh epidemiology of diabetes complications experience (1983-2005). Arch Intern Med 169: 1307-1316

2. Miller KM, Beck RW, Foster NC, Maahs DM (2020) HbA1c Levels in Type 1 Diabetes from Early Childhood to Older Adults: A Deeper Dive into the Influence of Technology and Socioeconomic Status on HbA1c in the T1D Exchange Clinic Registry Findings. Diabetes Technol Ther 22:645-650

3. Renard E, Ikegami H, Daher Vianna AG et al (2021) The SAGE study: Global observational analysis of glycaemic control, hypoglycaemia and diabetes management in T1DM. Diabetes Metab Res Rev. 37(7):e3430

4. Pickup J, Mattock M, Kerry S (2002) Glycemic control with continuous subcutaneous insulin infusion compared with intensive insulin injections in patients with type 1 diabetes: meta-analysis of randomized controlled trials. BMJ 324:705-708

5. Misso ML, Egberts KJ, Page M, OConnor D, Shaw J, (2010) Continuous subcutaneous insulin infusion (CSII) versus multiple insulin injections for type 1 diabetes mellitus. Cochrane Database Syst Rev. https://doi.org/10.1002/14651858.CD005103.pub2

6. Pickup JC, Sutton AJ (2008) Severe hypoglycaemia and glycaemic control in type 1 diabetes: meta-analysis of multiple daily insulin injections compared with continuous subcutaneous insulin infusion. Diabet Med 25:765-774

7. Foster NC, Beck RW, Miller KM et al (2019) State of Type 1 Diabetes Management and Outcomes from the T1D Exchange in 2016-2018. Diabetes Technol Ther 21:66-72

8. Retnakaran R, Hochman J, DeVries JH et al (2004) Continuous subcutaneous insulin infusion versus multiple daily injections. The impact of baseline A1c. Diabetes Care 27:2590-2596

9. Battelino T, Conget I, Olsen B et al (2012) The SWITCH Study Group. The use and efficacy of continuous glucose monitoring in type 1 diabetes treated with insulin pump therapy: a randomised controlled trial. Diabetologia 55:3155-3162

10. Bosi E, Choudhary P, de Valk HW et al (2019) The SMILE Study Group. Efficacy and safety of suspend-before-low insulin pump technology in hypoglycaemia-prone adults with type 1 diabetes 
(SMILE): an open-label randomised controlled trial. Lancet Diabetes Endocrinol 7:462-472

11. Danne T, Nimri R, Battelino $\mathrm{T}$ et al (2017) International consensus on use of continuous glucose monitoring. Diabetes Care 40:1631-1640

12. Boughton CK, Hovorka R (2021) New closed-loop insulin systems. Diabetologia 64:1007-1015

13. https://www.legifrance.gouv.fr/jorf/id/JORFTEXT000000458622

14. https://www.solidarites-sante.gouv.fr/IMG/pdf/diabecare_arrete_ et_cdc.pdf

15. Sherr JL, Tauschmann M, Battelino T et al (2018) ISPAD Clinical Practice Consensus Guidelines 2018: diabetes technologies. Pediatr Diabetes 19:302-325

16. Technology D (2020) Standards of Medical Care in Diabetes. Diabetes Care 43:S77-S88

17. Lassmann-Vague V, Clavel S, Guerci B et al (2010) When to treat a diabetic patient using an external insulin pump. Expert consensus. Société francophone du diabète (ex ALFEDIAM) 2009. Diabetes Metab 36:79-85

18. Roze S, Smith-Palmer J, Valentine W et al (2015) Cost-effectiveness of continuous subcutaneous insulin infusion versus multiple daily injections of insulin in Type 1 diabetes: a systematic review. Diabet Med 32:1415-1424
19. Gaudillière M, Pollin-Javon C, Brunot S, Villar Fimbel S, Thivolet C (2021) Effects of remote care of patients with poorly controlled type 1 diabetes included in an experimental telemonitoring programme. Diabetes Metab 47:101251

20. De Lagasnerie G, Aguadé AS, Denis P, Fagot-Campagna A, Gastaldi-Menager C (2018) The economic burden of diabetes to French national health insurance: a new cost-of-illness method based on a combined medicalized and incremental approach. Eur J Health Econ 19:189-201

21. Kawamura $\mathrm{T}$ (2007) The importance of carbohydrate counting in the treatment of children with diabetes. Pediatr Diabetes 8:57-62

22. Collyns OJ, Meier RA, Betts ZL et al (2021) Improved Glycemic Outcomes with Medtronic MiniMed Advanced Hybrid ClosedLoop Delivery: Results from a Randomized Crossover Trial Comparing Automated Insulin Delivery with Predictive Low Glucose Suspend in People with Type 1 Diabetes. Diabetes Care 44:969-975

Publisher's Note Springer Nature remains neutral with regard to jurisdictional claims in published maps and institutional affiliations. 